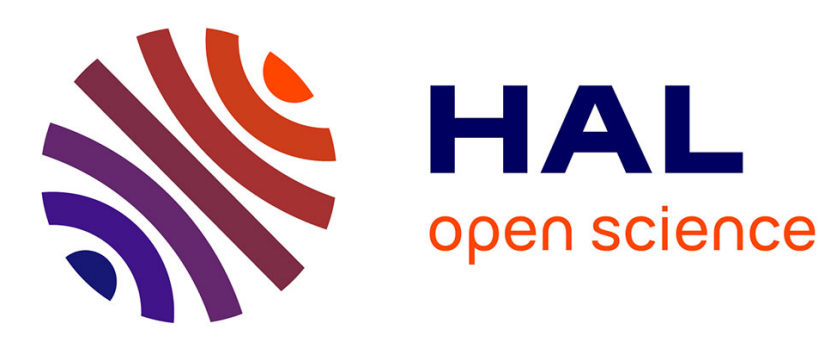

\title{
Possibilité, causalité et réquisits chez Hobbes et Leibniz Jean-Pascal Anfray
}

\section{To cite this version:}

Jean-Pascal Anfray. Possibilité, causalité et réquisits chez Hobbes et Leibniz. Paul Rateau; Eric Marquer. Leibniz, lecteur et critique de Hobbes, 21, Presses de l'Université de Montréal; Vrin, pp.77104, 2017, Analytiques, 978-2-7116-8420-5. hal-01611401

\section{HAL Id: hal-01611401 \\ https://hal.science/hal-01611401}

Submitted on 5 Oct 2017

HAL is a multi-disciplinary open access archive for the deposit and dissemination of scientific research documents, whether they are published or not. The documents may come from teaching and research institutions in France or abroad, or from public or private research centers.
L'archive ouverte pluridisciplinaire HAL, est destinée au dépôt et à la diffusion de documents scientifiques de niveau recherche, publiés ou non, émanant des établissements d'enseignement et de recherche français ou étrangers, des laboratoires publics ou privés. 


\title{
Possibilité, causalité et réquisits chez Hobbes et Leibniz
}

\author{
Jean-Pascal Anfray \\ École Normale Supérieure, République des Savoirs, centre Mathesis
}

(à paraître dans Paul Rateau et Eric Marquer (dir.), Leibniz, lecteur et critique de Hobbes, Montréal-Paris, Presses de l’Université de Montréal, Vrin, 2017, p.77-104).

\section{Introduction}

Le nécessitarisme est la thèse selon laquelle tout ce qui est actuel est absolument nécessaire ou, alternativement, tout ce qui est possible est actuel ${ }^{1}$. La nécessité en question est métaphysique, en sorte que le nécessitarisme exclut toute alternative possible à l'ensemble des choses et des événements actuels. Le nécessitarisme se distingue ainsi du déterminisme que l'on peut caractériser approximativement comme la thèse selon laquelle chaque état du monde est causalement nécessité par les états antérieurs. Le déterminisme est en effet compatible avec la possibilité d'états de choses inactuels, dans la mesure où il s'accorde avec la possibilité d'autres cours du monde, contrefactuels. Déterminisme et nécessitarisme sont donc deux thèses distinctes, ce qui n'empêche pas un philosophe de souscrire à la fois au déterminisme et au nécessitarisme. Ainsi Hobbes est à la fois déterministe et nécessitariste. Quant à Leibniz, il est très vraisemblablement déterministe, même si la nature de son déterminisme prête à discussion. Mais son rapport au nécessitarisme fait l'objet de débats parmi les commentateurs². L'intérêt d'aborder la

\footnotetext{
${ }^{1}$ Voir M. Griffin, Leibniz, God, and Necessity, Cambridge, Cambridge University Press, 2013, p. 58 et chap. 3 passim. Dans sa seconde formulation, le nécessitarisme coïncide avec le principe de plénitude. Il convient de noter que si la seconde caractérisation implique évidemment la première, l'implication réciproque est moins évidente car elle suppose l'incompatibilité de la nécessité absolue avec la possibilité du contraire.

${ }^{2}$ Parmi les commentateurs récents, M.Griffin est celui qui va le plus loin dans l'attribution à Leibniz d'une position nécessitariste ; cf. Griffin (2013), p. 67. Selon R. Adams, comme Griffin après lui, Leibniz se
} 
question du nécessitarisme chez Leibniz au prisme du rapport à Hobbes est précisément de mettre en avant la question du rapport entre nécessitarisme et déterminisme d'une part, et de comprendre d'autre part le rapport entre modalités logiques ou métaphysiques d'un côté et modalités causales de l'autre.

Dans la Théodicée, Leibniz estime que Hobbes déduit le nécessitarisme du déterminisme causal par un paralogisme reposant sur le glissement de la thèse selon laquelle toute possibilité exige une cause possible à l'affirmation que ce qui manque d'une cause actuelle est impossible. Nous verrons toutefois qu'aux yeux de Hobbes cette inférence n'est pas invalide, mais dépend de sa conception du possible fondée sur la notion de potentialité. En retour, nous nous intéresserons à la position ambivalente de Leibniz sur le rapport entre possibilité et réquisit causal. Contrairement à ce que laisse entendre la réfutation de Hobbes proposée dans la Théodicée, la réponse au nécessitarisme repose en effet sur une dissociation du possible métaphysique et du possible causal. Nous verrons également que cette dissociation est rendue possible par l'évolution de la conception de ce qu'est un réquisit, Leibniz passant d'une conception quasi-causale de ce qu'est un réquisit à une conception du réquisit comme ingrédient conceptuel, ce passage se produisant avec l'avènement de la définition de la vérité à partir de l'inhérence conceptuelle.

\section{Leibniz, Hobbes et le nécessitarisme}

La question du rapport de Leibniz au nécessitarisme est le plus souvent étudiée à travers le prisme des relations à Spinoza ${ }^{3}$, ce qui tend à occulter le rôle de Hobbes. Indéniablement, Spinoza représente pour Leibniz la figure principale lorsqu'il est question du nécessitarisme.

distingue de Hobbes et Spinoza dans la mesure où la nécessité en question est une nécessité morale, procédant d'un choix intelligent fondé sur l'idée de bien, à l'opposé de la nécessité brute d'un mécanisme aveugle ; voir R. M. Theist, Idealist, New York, Oxford University Press, 1994, chap.1, en particulier p. 20-22 et « Moral Necessity » in J. Cover et D. Rutherford (éds.), Nature and Freedom, New York, Oxford University Press, 2005, p. 181-193.

${ }^{3}$ Parmi les commentateurs récents, voir M. Lærke, Leibniz lecteur de Spinoza. Genèse d'une interprétation complexe, Paris, H. Champion, 2008; S. Newlands, «The Harmony of Leibniz and Spinoza », Philosophy and Phenomenological Research 81 :1 (2010), p. 64-104 ; M. Griffin (2013), p. 68-82. 
Dans le bref opuscule connu sous le titre « Deux sectes de naturalistes », rédigé vers la fin des années 1670, Leibniz rattache Spinoza à la secte des nouveaux Stoïciens qui affirment que Dieu agit avec une nécessité aveugle, tandis que Hobbes est associé au matérialisme d'Epicure et à la thèse selon laquelle tout, y compris Dieu, est composé de matière étendue ${ }^{4}$. Mais bien que Hobbes soit d'abord un représentant du matérialisme, il n'en reste pas moins une référence sur la question de la nécessité ${ }^{5}$. Ainsi, dans une lettre de 1680 adressée à Christian Philipp, Leibniz rattache au nécessitarisme de Spinoza et Hobbes la thèse cartésienne selon laquelle la matière reçoit successivement toutes les formes :

Ce sont justement les sentimens que Spinosa a expliqués plus clairement scavoir que justice, beauté, ordre, ne sont que des choses qui se rapportent à nous, mais que la perfection de Dieu consiste dans cette amplitude de son operation en sorte que rien ne soit possible ou concevable, qu'il ne produise actuellement. Ce sont aussi les sentimens de M. Hobbes qui soutient que tout ce qui est possible, est passé ou present ou futur (A II, $1^{2}, 787$ ).

Le nécessitarisme est présenté comme l'aboutissement conséquent de la thèse cartésienne $^{6}$. Dans le cas de Spinoza, Leibniz renvoie à la déduction à partir de la puissance divine. Il se contente pour Hobbes de relever l'adhésion de ce dernier au « principe de plénitude », selon lequel toute possibilité authentique doit être actuelle à un moment ou un autre ${ }^{7}$.

\footnotetext{
${ }^{4}$ A VI, 4-B, 1384-1385. La corporéité de Dieu est affirmée par Hobbes dans l'Appendice, chap.3 de l'édition latine du Léviathan, parue en 1668.

${ }^{5}$ Théod. §§ 171-173. Dans le compte-rendu de la controverse entre Hobbes et Bramhall figurant en appendice de la Théodicée, la question de la nécessité occupe une place centrale. Dans les passages de la Théodicée évoquant les partisans du nécessitarisme, Leibniz évoque outre Spinoza, les noms d'Abélard, Wyclif et Hobbes.

${ }^{6}$ Cf. Descartes, Principia Philosophiae, III, art. 47, AT, VIII-a, 103. La matière assume successivement toutes les formes possibles. Mais les formes en question sont des configurations spécifiques de la matière, non des configurations individuelles ou des corpuscules singuliers. Le principe de plénitude tel que le formule ce passage de Descartes n'implique donc pas la réalisation de toutes les possibilités individuelles.

${ }^{7}$ Voir A. O. Lovejoy, The Great Chain of Being, Cambridge, Harvard University Press, 1936. L'usage de ce principe dans l'étude des théories modales anciennes et médiévales a été systématisé par Jaakko Hintikka et
} 
Cela ne signifie pas pour autant que Hobbes serait une référence de moindre importance. En atteste le passage bien connu de l'opuscule « De la liberté » rédigé vers 1689, dans lequel Leibniz rappelle que c'est la considération des possibilités inactuelles qui lui permit d'échapper au nécessitarisme :

Mais je fus tiré de ce précipice par la considération de ceux des possibles qui ne sont pas, ne seront pas et n'ont pas été. Si en effet certains possibles n'existent jamais, alors les existences ne sont pas toujours nécessaires, sans quoi il serait impossible que d'autres existent à leur place et donc ceux qui n’existent jamais seraient impossibles («De libertate», A VI, 4B, 1653-54; TLM 329-30).

La contingence du monde actuel provient de la réalité de possibles inactuels, possibles dont les personnages de fictions romanesques, tels le roi Arthur, fournissent un modèle heuristique. Les lignes précédant immédiatement ce passage permettent d'identifier les motivations par lesquelles Leibniz aurait presque été conduit à adopter le nécessitarisme et l'on y retrouve des formules caractéristiques de l'influence de Hobbes :

Pour ma part, à l'époque où je considérais que rien ne se fait au hasard ou par accident, si ce n'est relativement à certaines substances particulières, que la fortune séparée du destin n'est qu'un nom vide de sens et que rien n'existe si ce n'est lorsque sont posés les réquisits singuliers (or il suit de ces réquisits, lorsqu'ils sont tous conjoints les uns aux autres, que la chose existe) ; j’étais peu éloigné de l'opinion de ceux qui jugent que tout est nécessaire, qu'il suffit que la liberté soit préservée de la contrainte bien qu'elle demeure soumise à la nécessité, et qui ne distinguent pas le nécessaire de l'infaillible, c'est-à-dire du vrai lorsqu'il est connu avec certitude (ibid.).

Leibniz a effectivement soutenu à un moment ou un autre chacune des thèses évoquées dans ces lignes. Dans ses premiers écrits, il soutient en particulier que la volonté et l'intelligence sont les conditions suffisantes pour la liberté de l'agent et l'absence d'obstacle définit alors la liberté d'action ${ }^{8}$. Dans une lettre à Wedderkopf de 1671, comme dans d'autres écrits du début de la même décennie, Leibniz conclut à la nécessité de toutes

Simo Knuuttila. Voir en particulier S. Knuuttila (éd.), Reforging the Great Chain of Being, Dordrecht, Londres, Reidel, 1981.

${ }^{8}$ A VI, 3, 133; A VI, 4-B, 1380; 1406. Dans ces textes, la contingence n'apparait pas comme une condition de la liberté, contrairement aux analyses ultérieures ; cf. Théod. §§ 288-290, 302. 
choses à partir du principe de raison suffisante et de la raison suffisante conçue comme agrégat de réquisits 9 .

Bien que Hobbes ne soit pas cité ni évoqué nommément, chacune de ces thèses semble bien témoigner de l'influence de celui-ci. La négation de la fortune et du hasard est en effet un aspect important de la controverse avec Bramhall ${ }^{10}$. Davantage, la liberté, définie comme absence de contrainte, renvoie à la définition de la liberté comme absence d'obstacle de Hobbes ${ }^{11}$. Un indice plus décisif encore de l'origine hobbesienne du nécessitarisme initial tient à l'usage du concept de réquisit dans la formulation du nécessitarisme. Le nécessitarisme est en effet présenté ici comme déduit du principe de causalité et de la notion de réquisit, compris comme condition nécessaire de l'existence d'une chose. La cause suffisante peut ainsi être définie comme un ensemble ou un agrégat de réquisits. Cette notion de réquisit, comprise comme une condition d'existence, est en effet utilisée par Leibniz dans un certain nombre de textes des années 1670. La ratio ou la causa est définie comme un agrégat de tous les réquisits d'une chose ${ }^{12}$. C'est le cas en particulier dans un passage du De summa rerum de 1676 :

\footnotetext{
${ }^{9}$ À Wedderkopf, mai 1671, A II, 1, 186. La nécessité dépend toutefois de 1'harmonie et la perfection intrinsèque. Le nécessitarisme de la lettre à Wedderkopf est essentiellement axiologique, en quoi il diffère profondément du nécessitarisme mécaniste de type hobbesien.

${ }^{10}$ Sur le hasard, voir $Q L N C$, Foisneau-Perronin 45. Le fortuit ou contingent sont des modalités purement épistémiques, relatives à notre degré d'ignorance des causes (DCo, X.5). Leibniz approuve le refus hobbesien du hasard, cf. Théod., Réfl. sur Hobbes, § 2, GP VI, 389. Dans la tradition scolastique, le hasard n'est pas une cause par soi, mais accidentelle, lorsqu'une cause agit hors du cours naturel des choses. Il n'a de réalité qu'au point de vue des causes particulières, mais disparaît au point de vue de Dieu et de sa providence. Cf. Thomas d'Aquin, Summa Theologiae (= Sum. Theol.), I, q. 22, a. 2, ad 1; Suárez, Disputationes Metaphysicae (= Disp. Met.). XIX, sec. 12, n. 5, in Opera omnia, éd. C. Berton, Paris, L. Vivès, 1856-1861, vol. xxv, p. 743 b.

${ }^{11}$ Sur le refus de la liberté opposée à la nécessité, cf. DCo, XXV.13 (OL, I, 333). Sur la liberté comme absence d'obstacle et de contrainte extérieure, cf. QLNC, XXIX, Foisneau- Perronin 348 ; Dho, XI, 2 (OL, II, 95).

12 12. Cf. A VI, 2, 483; A VI, 3, 118; 133: « Si quid non existit, requisitum profecto aliquod deesse necesse est, quia nihil aliud est definitio, quam enumeratio requisitorum »; 474 : « Ratio rerum, aggregatum requisitorum omnium rerum »; $573:$ «Ultima ratio est aggregatum requisitorum sufficientium »; 584 « Nihil
} 
Il est nécessaire pour l'existence que soit présent l'agrégat de tous les réquisits. Un réquisit est ce sans quoi la chose ne peut pas être (id sine quo res esse non potest). L'agrégat de tous les réquisits est la cause pleine de la chose. Rien n'est sans raison. Car rien n'est sans l'agrégat de tous les réquisits [...]. Pour un corps donné, l'agrégat de tous ses réquisits est en dehors des corps. L'agrégat de tous les réquisits d'un corps et l'agrégat de tous les réquisits d'un autre corps sont dans un seul et même être. Cet Être unique, quel qu'il soit, est la raison ultime des choses (A VI, 3, 587, TLM 30).

Pour un être fini, ici un corps quelconque, comme pour un ensemble d'êtres finis, un ensemble de corps, l'agrégat de tous leurs réquisits qui forme la raison suffisante de leur existence est extérieur et unique. En outre, aucun être ne peut exister sans que soit donné l'agrégat de tous ses réquisits. Une chose ne peut exister sans l'un de ses réquisits. Dans cette formule, la portée de la modalité exprimée par le verbe potest est ambiguë et peut en effet se prêter à une lecture nécessitariste si on comprend qu'une chose est impossible lorsqu'un de ses réquisits lui fait défaut. Une telle formule, reprise dans le De libertate, ouvre la voie à un argument nécessitariste inspiré de Hobbes.

Cette voie d'entrée dans le nécessitarisme est distincte de celle de Spinoza. Le nécessitarisme chez Spinoza est en effet tiré de la considération de la nature divine, en particulier de sa puissance infinie. Chez Hobbes en revanche, le nécessitarisme est établi à partir de l'analyse des réquisits causaux d'un événement ou de l'existence d'une chose. L'argument repose sur l'idée qu'il n'y a pas de possibilité sans une puissance, un pouvoir ou encore une capacité susceptible de produire l'état de choses considéré comme possible. Il s'agit d'un modèle du possible comme puissance ou potentialité. L'adoption d'un tel modèle du possible ne débouche pas automatiquement sur le nécessitarisme mais peut toutefois y conduire, selon les conditions d'attribution d'un pouvoir ou d'une capacité à un sujet, et il y a des antécédents historiques, comme Avicenne, ou, plus lointainement, les

est sine causa, quia nihil est sine omnibus ad existendum requisitis ». Cette notion de la raison pleine comme agrégat de tous les réquisits se retrouve dans des textes ultérieurs, par exemple dans le Specimen inventorum, A VI, 4-B, 1618. Adams (1994, p. 117-118) soutient que les deux relations de dépendance, causale et conceptuelle, fusionnent chez Leibniz. S. Di Bella, au contraire, insiste sur la distinction ultérieure chez Leibniz entre dépendance causale et conceptuelle ; cf. S. Di Bella, The Science of the Individual. Leibniz’s Ontology of Individual Substance, Springer, 2005, p. 78 sq. Nous verrons plus loin que la confrontation avec Hobbes et la conception du possible défendue par Leibniz infirment la lecture d'Adams. 
Mégariques $^{13}$. Les manuels de philosophie du $\mathrm{xvi}^{\mathrm{e}}$ siècle ont contribué à répandre, tout en la critiquant, cette version causale du nécessitarisme ${ }^{14}$, que le jésuite Benedictus Pererius décrit ainsi :

[U]n agent naturel, s'il possède tout ce qui est nécessaire à l'action ne peut pas ne pas agir ; de même s'il manque l'une de ces choses nécessaires, il ne peut pas agir ; c'est pourquoi, lorsqu'il agit, il agit nécessairement, et s'il n'agit pas, c'est parce qu'il ne peut pas alors agir; c'est pourquoi s'il produit un effet, il le produit nécessairement, et s'il ne le produit pas, alors il y a nécessairement eu un empêchement à ce qu'il le produise. Donc, bien que selon Aristote les choses sublunaires n'arrivent pas par nécessité, contrairement aux choses célestes, étant donné qu'elles n'arrivent pas toujours de la même façon et qu'il leur arrive d'être déficientes par rapport à leur ordre et de leur cours naturel, néanmoins, de ce que lorsqu'elles arrivent, elles arrivent nécessairement, et lorsqu'elles sont déficientes, elles sont nécessairement déficientes, en ce sens on peut dire qu'elles arrivent par nécessité. Avicenne a soutenu cette opinion dans sa Métaphysique, comme le rapporte Thomas dans la Somme contre les Gentils III, chap. 86, et Ockham la suit dans son Quodlibet I, qu.17 (B. Pererius, De communibus omnium rerum naturalium principiis et affectionibus, lib. IX, cap. 4, Paris, 1579, p. $515)$.

Dans les choses naturelles, la contingence n'a qu'un sens statistique, correspondant au fait que des effets d'un certain type ne se produisent pas toujours dans des conditions données. En revanche, un effet singulier, par exemple une flamme embrasant une torche, a

\footnotetext{
${ }^{13}$ Pour les Mégariques, Aristote, Métaphysique IX, 3, 1046b29-36. Thomas d'Aquin attribue à Avicenne une position nécessitariste (cf. Sum. Theol. I, q. 115, a. 6, in corp.). Mais c'est Averroès qui est vraisemblablement responsable de l'attribution d'une position nécessitariste, en prêtant à Avicenne l'idée que l'existence d'une cause est une condition de la possibilité même du possible (cf. Destructio destructionum, in Opera Omnia Aristotelis cum Averrois Commentariis, Venise, ap. Iuntina, 1562-1574, vol. IX, f. 71K-L). Cela conduirait effectivement au nécessitarisme : si à aucun moment du temps il n'y avait une cause d'une maison heptagonale, celle-ci ne serait pas possible et réciproquement, tout ce qui est possible existerait par une cause à un moment ou un autre. Voir la mise au point d'A. Bäck, « Avicenna and Averroes : Modality and Theology », in C. H. Kneepkens, K. Lorenz (éds.), Potentialität und Possibilität. Modalaussagen in der Geschichte der Metaphysik, Frommann-Holzboog, 2001, p. 125-146.

${ }^{14}$ Cf. A. Maier, Die Vorläufer Galileis im 14. Jahrhundert. Studien zur Naturphilosophie der Spätscholastik, Rome, Edizioni di Storia e Letteratura, 1949, p. 241-250; C. Leijenhorst, The Mechanisation of Aristotelianism: The Late Aristotelian Setting of Thomas Hobbes's Natural Philosophy, Leyde, Brill, 2002, p. 183-185.
} 
nécessairement lieu si les réquisits sont posés, en particulier si l'agent (la flamme) et le patient (la torche) sont suffisamment rapprochés et s'il y a une quantité d'air suffisante dans la pièce. Si l'effet ne se produit pas, c'est soit parce qu'un réquisit faisait défaut - la flamme était trop éloignée, par exemple, ou il manquait d'air - soit en raison d'un empêchement - par exemple, la torche avait été plongée dans l'eau auparavant. L'agent, la flamme, ne peut donc embraser la torche dans ces circonstances et il est absolument impossible que cette torche puisse brûler à ce moment. En généralisant, si la cause d'un effet singulier n'est jamais donnée, celui-ci est absolument impossible. Il n'y a pas de distinction entre cause suffisante et nécessaire, dans la mesure où les empêchements - ou plus exactement l'absence de ces empêchements - sont une partie de la cause suffisante elle-même. Il convient cependant de préciser que les scolastiques dont Pererius rapporte qu'ils ont soutenu cette thèse, Ockham en particulier, restreignent cette analyse aux agents naturels, mais maintiennent une contingence réelle pour les effets issus d'agents libres ${ }^{15}$. Ces derniers ont en effet le pouvoir, étant donné l'ensemble des réquisits, de vouloir une action et de ne pas la vouloir. Par conséquent, ce qu'un agent libre ne veut pas n'est pas impossible pour autant.

Hobbes radicalise l'argument nécessitariste tiré de la considération de la causalité, en identifiant purement et simplement les notions de cause entière, cause suffisante et cause nécessaire et le généralise en l'étendant à tous les êtres sans exception. Cette équivalence apparaît clairement dans le De Corpore :

La cause entière suffit toujours pour produire son effet, pourvu que l'effet soit absolument possible.

Car quelque effet à produire que l'on propose, s'il se produit, il est manifeste que la cause qui l'a produit a été suffisante. S'il n'a pas été produit, et qu'il fut cependant possible, il est clair qu'il a manqué quelque chose, soit dans un agent soit dans le patient, sans lequel il n'a pu se produire, autrement dit, qu'il a manqué un accident qui est un réquisit de sa production. C'est pourquoi cette cause n'était pas entière, contre la supposition (DCo, IX, 5, OL, I, 108).

\footnotetext{
${ }^{15}$ Cf. Guillaume d'Ockham, Quodlibeta septem, éd. J.C. Wey, Opera theologica vol. IX, New York, St Bonaventure, 1981, p. 90-91.
} 
Un exemple emprunté à la controverse avec Bramhall illustre ce raisonnement ${ }^{16}$. Une voiture a besoin d'être tirée par deux chevaux pour rouler. Si la voiture roule, c'est qu'elle possède une cause suffisante. Cette cause suffisante est composée de l'ensemble des réquisits : non seulement la présence de deux chevaux, mais encore leur bonne santé, leur dressage correct, le fait qu'ils soient correctement attelés, que la voiture soit en état de rouler, etc. ${ }^{17}$ L'ensemble de ces réquisits forme la cause entière. Mais il suffit que tous ces réquisits soient posés pour que la voiture roule. La cause entière est donc la cause suffisante. La cause entière est alors l'agrégat de tous les réquisits qui nécessite l'occurrence de l'effet :

C'est pourquoi la cause de tous les effets consiste dans des accidents déterminés des agents et du patient, tels que, lorsqu'ils sont tous présents, l'effet se produit, si l'un d'eux est absent, il ne se produit pas. Or l'accident soit de l'agent soit du patient sans lequel un effet ne peut se produire s'appelle cause sine qua non, nécessaire par hypothèse et réquisit de la production de l'effet. Et la cause absolument parlant ou cause entière (causa integra) est l'agrégat de tous les accidents tant des agents, aussi nombreux soient-ils, que du patient, tels que s'ils sont tous supposés on ne peut entendre (intelligi non potest) que l'effet ne se produise pas et tels que, supposé que l'un d'eux soit absent, on ne peut entendre que l'effet se produise (DCo, IX, 3, OL, I, 107-108).

En l'absence de l'un de ces réquisits, non seulement l'effet ne se produit pas, mais en outre il est inconcevable qu'il se produise, ce qui permet ainsi de conclure à son impossibilité absolue. En effet pour qu'un effet puisse se produire, il est nécessaire que ses

\footnotetext{
${ }^{16}$ QLNC, XXXI, Foisneau-Perronin 358-361.
}

${ }^{17}$ En réalité, comme l'a justement remarqué L. Foisneau, la cause suffisante de n'importe quel effet, d'une part, remonte jusqu'à la cause première, d'autre part, doit envelopper la totalité des choses faisant partie de l'univers. C'est ce qu'il appelle le «paradoxe de Hobbes », selon lequel «un effet ne peut avoir une cause suffisante... si l'un des agents dont est fait l'univers ne concourt pas à le produire... Tout ce qui est in rerum natura contribue à produire l'effet, fût-il en apparence le plus fortuit qui soit ». Voir L. Foisneau, Hobbes et la toute-puissance de Dieu, Paris, Presses Universitaires de France, 2000, p. 119 et Hobbes, QLNC, XXI, p. 294. Il faut ajouter que Hobbes se distingue en outre de la tradition scolastique en identifiant la cause non à une substance active, déterminée par un ensemble de réquisits, mais à un agrégat de réquisits, autrement dit d'accidents des corps, qui ne sont rien d'autre que des mouvements et leurs déterminations. 
réquisits soient présents, ce qui par hypothèse n'est pas le cas pour un effet inactuel ${ }^{18}$. Ainsi, Hobbes conclut à l'identité de la cause suffisante et de la cause nécessaire et affirme que ce qui arrive est nécessité par des causes antécédentes ${ }^{19}$.

\section{La réfutation leibnizienne de la position de Hobbes dans la Théodicée}

Leibniz revient au nécessitarisme de Hobbes dans la Théodicée. D’après lui, la déduction de Hobbes repose sur un paralogisme, identifié et réfuté au $§ 172$ de l'ouvrage :

De nos jours, le fameux M. Hobbes a soutenu cette même opinion, que ce qui n'arrive point est impossible. Il la prouve, parce qu'il n'arrive jamais que toutes les conditions requises à une chose qui n'existera point (omnia rei non futurae requisita) se trouvent ensemble: or la chose ne saurait exister sans cela. Mais qui ne voit que cela ne prouve qu'une impossibilité hypothétique ? Il est vrai qu'une chose ne saurait exister, quand une condition requise y manque. Mais comme nous prétendons pouvoir dire que la chose peut exister, quoiqu'elle n'existe pas, nous prétendons de même pouvoir dire que les conditions requises peuvent exister, quoiqu'elles n'existent point. Ainsi l'argument de M. Hobbes laisse la chose où elle est (Théodicée, § 172).

Le paralogisme de Hobbes repose sur un simple glissement de la portée de l'opérateur modal d'impossibilité. Toutefois il est utile de reconstruire l'argument de Leibniz. L'expression « chose qui n'existera point » est relativement indéterminée et recouvre une pluralité de cas. Il peut s'agir de substances individuelles simplement possibles, comme le roi Arthur, mais également d'événements possibles, comme la mort de Spinoza à Leyde, selon l'exemple emprunté à Bayle (Théod. §174). Un événement correspond à ce que Leibniz appelle ailleurs un état d'une chose (status rei, cf. A VI, 4-A, 569), c'est-à-dire à une proposition énonçant un prédicat contingent au sujet d'une substance. Mais il peut encore s'agir d'un terme ou concept ou encore des propositions contenant ces concepts, qu'il s'agisse de propositions d'existence ou de propositions prédicatives. Leibniz

\footnotetext{
${ }^{18}$ Cf. $D C o, \mathrm{X}, 4, O L, \mathrm{I}, 115$ : «si potentia plena nunquam erit, semper deerit aliquod eorum, sine quibus produci actus non potest ; actus ergo ille nunquam produci poterit, id est, actus ille impossibilis est ».

${ }^{19}$ DCo, IX.5, OL, I, 109 : «... [Q]uicunqueunquameffectusfuturisunt,causamneces- sariam habituros esse, atque hoc modo quaecunque producta vel erunt vel fuerunt, necessi- tatem suam in rebus antecedentibus habuisse ».
} 
considère en effet les modalités tantôt comme des propriétés de concepts ou termes incomplexes, tantôt comme des prédicats de propositions ou termes complexes ${ }^{20}$. Afin de simplifier la reconstruction, on considérera des propositions singulières notées $p$ et un opérateur modal $M$ correspondant à l'idée de possibilité absolue, en tant que cette possibilité se distingue conceptuellement de la possibilité physique ou causale, qui est incompatible avec la nécessité hypothétique ${ }^{21}$ :

(NEC) $\neg p \supset \neg M p$ (« ce qui n'arrive point est impossible »)

Afin d'établir (NEC), Hobbes recourt comme on l'a vu à la notion de réquisit et à celle d'agrégat de réquisits. Soit $r$ l'agrégat des réquisits de $p$. D'après la reconstruction proposée par Leibniz, le raisonnement de Hobbes est le suivant :

1. $\neg \mathrm{M}(r \& \neg p)$ (« il n'arrive jamais que toutes les conditions requises à une chose qui n'existera point [omnia rei non futurae requisita] se trouvent ensemble »)

2. $\neg \mathrm{r} \supset \neg \mathrm{Mp}(\ll$ la chose ne saurait exister sans cela »)

La prémisse (1) résulte de la définition de la cause entière comme l'agrégat de tous les réquisits d'une chose. Chaque réquisit est en quelque sorte une condition nécessaire mais insuffisante, tandis que l'agrégat des réquisits est la condition suffisante. Si une chose n'existe pas actuellement, il lui manque une condition suffisante actuelle. Dans cette hypothèse, l'agrégat de ses réquisits ne peut être donné. Leibniz concède la vérité de (1). Cependant, il diagnostique un glissement erroné de la portée de l'opérateur modal dans (2), de l'impossibilité de la conséquence à l'impossibilité du conséquent. (2) permet effectivement de déduire (NEC) à partir de (1). Mais (2) est fausse ou du moins dépourvue d'évidence. L’idée qu'une chose « ne saurait exister » sans l'ensemble de ses réquisits devrait être formulée ou bien ainsi :

\footnotetext{
${ }^{20}$ Voir en particulier A VI, 4-A, 749 ; 758-759.

${ }^{21}$ Une proposition en ce sens est impossible si elle implique contradiction, possible si elle n'implique pas contradiction, nécessaire si elle peut être résolue à une identique ou si la proposition contraire implique contradiction. Plus précisément, une proposition «A est B » est impossible si elle est démonstrativement réductible (i.e. en un nombre fini d'étapes par analyse des termes du sujet et du prédicat) à une contradiction de la forme « A est non-A ». Cf. A VI, 4-A, 758.
} 


$$
2^{\prime} \cdot \neg M(p \& \neg r)
$$

Ou bien, de manière équivalente (où $L$ signifie « il est absolument nécessaire que ») :

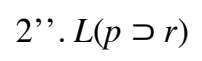

Or ni (2') ni (2'’) ne permettent de déduire (NEC), à moins que l'impossibilité de $r$ n'ait été établie indépendamment ${ }^{22}$. (NEC) ne peut donc être validement déduit de (1) et (2') ou (2'). Ainsi, Hobbes ne conclut à la nécessité absolue que parce qu'il suppose indûment que la simple absence d'une cause actuelle d'un événement possible, par exemple de la mort de Spinoza à Leyde, équivaut à l'impossibilité d'une cause actuelle de cet événement, entraînant du même coup l'impossibilité absolue de la mort de Spinoza à Leyde. Or l'absence d'une cause entière permet au plus de conclure à l'impossibilité hypothétique de cet événement : «Ainsi l'argument de M. Hobbes laisse la chose où elle est ». Hobbes aurait confondu la nécessité causale, simplement hypothétique (celle de 2' et 2',), avec la nécessité métaphysique absolue de (NEC).

La reconstruction de la réfutation de Leibniz révèle également un aspect de sa propre conception du possible. La possibilité d'une chose est certes indépendante de l'existence actuelle de la totalité de ses réquisits ; elle implique toutefois leur possibilité. Autrement dit, la possibilité de cette chose est fondée dans la possibilité de ses réquisits, ce qui peut se formuler ainsi :

(POS/R) La possibilité métaphysique de $p$ implique la possibilité de l'en- semble des réquisits de $p$ (i.e. $L(M p \supset M r)$ ).

L'adhésion de Leibniz à (POS/R) est importante en vertu de sa converse qui établit en particulier que si un état de choses est tel qu'il n'y a nulle cause possible en vertu de laquelle il peut avoir lieu, cet état de choses est impossible. En outre, puisqu'un réquisit désigne une cause et l'ensemble des réquisits une cause suffisante, cela signifie que la possibilité absolue d'une chose dépend de la possibilité d'une cause suffisante de cette chose. Il n’y aurait pas de possibilités pures, « a-causales » pour ainsi dire. Avant de revenir

\footnotetext{
${ }^{22}$ Supposons en effet l'impossibilité de $r$. D'après (2'), $p$ impliquerait alors une impossibilité. Or la possibilité est close sous l'implication. Autrement dit, si $p$ et possible et si $p$ implique $q$, alors $q$ est possible.
} 
à la question de savoir si Leibniz souscrit effectivement à (POS/R), il convient de se pencher sur le rapport de Hobbes au nécessitarisme.

\section{Hobbes : puissance totale et possibilité}

Selon Leibniz, Hobbes est partisan du nécessitarisme et l'établit par un paralogisme. Leibniz ne fait pas erreur sur le premier point. L'adhésion de Hobbes au principe de plénitude ne fait aucun doute. Un acte ou un événement possible est ainsi défini comme celui qui se produit à un moment du temps ${ }^{23}$. Réciproquement, un événement qui ne se produit pas est impossible. Tout événement est nécessaire ou impossible et la possibilité d'un événement, loin d'exclure la nécessité absolue de son occurrence, la présuppose au contraire. La nécessité qui s'attache aux événements n'est pas moindre que la nécessité d'une vérité essentielle comme "l'homme est un homme ${ }^{24}$. Ainsi la nécessité causale n'est pas une forme affaiblie de nécessité, distincte de la nécessité logique.

La contingence repose seulement sur notre ignorance des causes. Un énoncé singulier portant sur le futur, comme « il pleuvra demain » que l'on suppose vrai, est qualifié de futur contingent parce que nous ne concevons qu'une partie de la cause totale de cet événement, compatible avec le fait qu'il ne pleuvra pas demain. Si nous connaissions ces causes, nous jugerions que cet énoncé est nécessaire ou impossible ${ }^{25}$. La contingence ne réside que dans notre manière de concevoir les choses.

${ }^{23} \mathrm{DCo}, \mathrm{X}, \S 4,115$ : « actus omnis possibilis aliquando producetur ».

${ }^{24}$ DCo, X, $\S 5,115:$ : [...] omnis actus possibilis aliquando producetur. Imo vero non minus necessaria propositio est Futurum est futurum quam homo est homo ». À l'encontre de la lecture de Leijenhorst (2002), p. 186. L. Foisneau défend une interprétation nécessitariste ; cf. Foisneau (2000), chap. 3 passim. Dans la controverse avec Bramhall, Hobbes défend la doctrine de la nécessité contre l'objection selon laquelle les événements ne sont nécessaires qu'en vertu d'une nécessité hypothétique, consécutive à la supposition de l'événement.

${ }^{25}$ DCo, $\mathrm{X}, \S 5,116$ : «Propositio itaque omnis de futuro contingente vel non contingente, qualis est haec Cras pluet vel haec Cras Sol orietur, necessario vera est vel necessario falsa. Sed quoniam, utrum vera an falsa sit, nondum scimus, ideo vocamus eam contingentem [...]». Sur la critique hobbesienne des futurs contingents, voir L. Foisneau, « De la nécessité des choses et des actions. Hobbes critique de l'indétermination des futurs 
La nécessité et l'impossibilité dépendent elles aussi de nos capacités de conception. Or cette thèse pourrait sembler affaiblir le nécessitarisme. Un énoncé nécessaire, par exemple « l’homme est un animal », est défini comme un énoncé tel que nous ne pouvons concevoir aucune circonstance dans laquelle le nom sujet pourrait s'appliquer à une chose sans que le nom figurant comme prédicat ne puisse s'appliquer à cette chose ${ }^{26}$.

Selon Hobbes, le possible et l'impossible sont fondés dans nos capacités de conception :

Parce qu'il (quia) n'est pas possible de concevoir si le monde est, qu'il ne soit monde, ou si l'homme est, qu'il ne soit animal [...], pour cette raison (ideo) de telles propositions sont appelées nécessaires ; car ce que nous ne pouvons concevoir se faire autrement (aliter se habere), nous le disons nécessaire. Donc ce ne pas pouvoir concevoir autrement n'est pas une incapacité des choses, mais la nôtre... $(A W$, XXVIII, $\S 7,337)$.

La même réduction de la nécessité à l'inconcevabilité du contraire se retrouve dans la définition de la cause entière citée plus haut. La philosophie première justifie cette réduction de l'ensemble des modalités à des modes de représentation. Hobbes identifie en effet possibilité, puissance et cause. Une cause est à son tour définie comme une somme ou un agrégat d'accidents. Or les accidents sont définis comme des « modes de concevoir les corps » (DCo, VIII, $\S 2,92)$. Les accidents désignent les différentes façons dont les corps nous affectent et nos manières de les concevoir ${ }^{27}$. C'est pourquoi le possible et l'impossible coïncident avec les limites de nos représentations. Dans la mesure où Dieu est la cause première de nos représentations, la distinction du possible et de l'impossible, loin de définir les limites de la toute-puissance divine, est instaurée par cette toute-puissance ${ }^{28}$. Mais si la

contingents », in L. Foisneau (éd.), La Découverte du principe de raison. Descartes, Hobbes, Spinoza, Leibniz, Paris, PUF, 2001, p. 91-118.

${ }^{26}$ DCo, III, § 7, 33 : «Necessaria [propositio] est, quando nulla res concipi potest sive fingi ullo tempore, cujus nomen sit subjectum, quin ejusdem nomen sit etiam praedicatum ».

${ }^{27}$ Sur la question du statut de l'accident, voir Martine Pécharman, « Le vocabulaire de l'être dans la philosophie première : ens, esse, essentia », in Hobbes et son vocabulaire. Études de lexicographie philosophique, Yves-Charles Zarka (dir.), Paris, Vrin, 1990, p. 31-59 et dans ce volume, p. 114-115.

${ }^{28}$ Voir M. Pécharman, «La puissance absolue de Dieu selon Hobbes » in Potentia Dei, L'onnipotenza nel pensiero dei secoli XVI e XVII, G. Canziani, M.A. Granada, Y.-C. Zarka, Milan, Franco Angeli, p. 269-293, à la p. 280. 
toute-puissance n'est pas soumise aux limites du possible, cela semble invalider le nécessitarisme. Cela supposerait de penser la toute-puissance comme un pouvoir s'étendant à du créable débordant la création actuelle. Or Hobbes rejette une telle conception de la toute-puissance et soutient au contraire qu'il n'y a pas de pouvoir en Dieu de faire ce qu'il ne veut pas à un moment ou un autre. Ainsi, la considération de la toute-puissance divine n'invalide pas le nécessitarisme mais en constitue au contraire le fondement théologique.

Hobbes aurait-il dû cependant remarquer le paralogisme que Leibniz pense déceler dans son argument établissant l'identité de la cause entière et de la cause nécessaire, autrement dit la confusion entre l'impossibilité hypothétique issue de l'absence d'une cause actuelle et l'impossibilité absolue résultant de l'absence de cause possible ? Rien n'est moins sûr. En effet, la thèse de Hobbes repose sur une conception du possible comme potentialité et sur une condition stricte sur l'existence d'une puissance.

Ainsi au chapitre $\mathrm{X}$ du De Corpore, la distinction de la puissance et de l'acte est reconduite à la distinction de la cause et de l'effet, ces deux couples de distinctions ne différant que par la connotation temporelle. Hobbes définit ainsi la puissance respectivement active et passive comme l'ensemble des réquisits de la cause efficiente et matérielle avant la production de l'effet. Il ajoute :

L'acte impossible en effet, est celui pour lequel il n'y aura jamais une puissance pleine pour le produire. Mais puisque la puissance pleine est celle dans laquelle concourt tout ce qui est requis pour produire l'acte, si la puissance n'est jamais pleine, il manquera toujours l'un de ce sans quoi l'acte ne peut être produit ; donc cet acte ne pourra jamais être produit, autrement dit cet acte est impossible. Mais l'acte qui n'est pas impossible, est possible. Aussi tout acte possible se produira à un moment. Car s'il ne se produit jamais, jamais ne concourra tout ce qui est requis à sa production ; c'est que cet acte est impossible (par définition), ce qui est contre la supposition (DCo, X, 4, OL, I, 115).

La puissance pleine est la conjonction de la puissance active et passive et elle est identique à la cause entière. Or la cause entière est simultanée avec l'effet, ou plus précisément le dernier réquisit qui fait que la cause est entière est simultané à l'effet ${ }^{29}$. La

\footnotetext{
${ }^{29}$ DCo, IX. 5, OL, I, 109 : «Ex quo et hoc sequitur: quo instante causa fit integra, eodem quoque effectum esse productum. Nam si non sit productus, deest adhuc aliquod ad produc- tionem requisitum ; non erat ergo, ut supponebatur, causa integra ».
} 
puissance pleine coïncide donc avec l'acte. Toute puissance pleine étant actualisée, il s'ensuit qu'il n'y a pas de puissance totale avant l'acte lui-même, mais seulement des puissances partielles. Ainsi il faut dire que le cheval peut tirer la voiture s'il est correctement attelé à un autre cheval et s'il est bien dressé ou encore si la voiture est en état de rouler, etc. Le cheval pris isolément ou plus exactement sa capacité à mouvoir correspond à une puissance partielle de l'événement qu'est la traction de la voiture. Dans la définition de l'agent libre figure un élément conditionnel : « un agent libre est celui qui peut faire s'il le veut et s'abstenir s'il le veut ${ }^{30} »$. Cet élément conditionnel désigne lui aussi une puissance partielle. Les opportunités et l'absence d'empêchement constituent des puissances partielles pour qu'un agent accomplisse telle ou telle action. La puissance totale suppose le concours de ces puissances partielles.

Une puissance partielle, définie comme un sous-ensemble de réquisits d'un effet donné, c'est-à-dire comme une puissance active ou passive, n'est donc pas une puissance simpliciter, mais seulement une puissance conditionnelle et l'acte correspondant n'est pas constitué en possibilité réelle ${ }^{31}$. Inversement, seule la puissance totale qui coïncide avec la cause entière définit une possibilité réelle ${ }^{32}$. Or nous avons vu que la cause entière est

\footnotetext{
${ }^{30}$ QLNC, XXXIII, p.366.

${ }^{31}$ DCo, X.3, OL, I, 115: « ideoque potentiae illae, posse scilicet agens, si modo patienti applicetur, et posse patiens, si modo agenti applicetur ; aliter neutrum quicquam posse, neque ergo accidentia, quae in illis sunt, potentiae proprie loquendo dici possunt neque actus aliquis possibilis dici propter potentiam solius agentis aut solius patientis » (nous soulignons).

${ }^{32} \mathrm{Si}$ l'on ajoute que la totalité des conditions nécessaires est une condition suffisante de l'effet, alors la possibilité coïncide avec l'actualité à un moment du temps. Pour autant, Hobbes ne soutient pas que la possibilité se restreint au moment où la puissance est pleine. Si un effet $e$ se produit à $t$, c'est qu'il y a une puissance pleine à $t$ pour que l'effet se produise à $t$. Mais dès lors qu'il y a une puissance pleine, $e$ est possible à n'importe quel autre moment. La définition de la possibilité totale comme ensemble des conditions nécessaires revient à N. Hartmann. Cf. N. Hartmann, « Der megarische und der Aristotelische Möglichkeitsbegriff » (1937), in Kleinere Schriften II, Berlin, De Gruyter, 1957, p. 85-100 et H. Weidemann, « Aristotle, the Megarics, and Diodorus Cronus on the Notion of Possibility », American Philosophical Quarterly 45.2 (2008), p. 131-148.
} 
simultanée avec son effet. Cela signifie que la puissance totale coïncide avec l'acte, en sorte que, nécessairement, toute possibilité réelle est actuelle à un moment ou un autre du temps.

Imaginons que Bramhall ait effectivement rédigé sa réplique, il est alors impossible qu'il ne l'ait pas écrite, car il manque un réquisit dans l'agrégat qui constituerait la cause suffisante (par hypothèse absente) de sa non-rédaction. Plus généralement, celui qui délibère l'ignore encore et peut ainsi se figurer des scénarios alternatifs selon la décision qu'il adoptera, mais ces scénarios ne correspondent qu'à de simples possibilités épistémiques ${ }^{33}$.

Les hypothèses physiques ont un statut analogue, mais introduisent une difficulté pour la définition de la cause entière. En effet, la cause entière d'un effet est telle que si un seul des réquisits est manquant, on ne peut concevoir que l'effet se produise. L'incapacité de concevoir fonde, comme on l'a vu, l'impossibilité absolue de l'effet. Or, selon Hobbes, dans la physique ou science des phénomènes de la nature, nous pouvons toujours concevoir plusieurs hypothèses explicatives alternatives à un même effet, car les phénomènes sousdéterminent la théorie correcte. Ainsi, au début du chapitre XXV du De Corpore, Hobbes indique qu'à partir des phénomènes nous pouvons déterminer les causes possibles de la génération. Plusieurs causes semblent donc possibles (en tant que possibilités épistémiques). Cependant, les explications fondées sur ces hypothèses n'ont pas valeur de démonstration à proprement parler, mais sont adoptées en vertu de leur pouvoir explicatif (XXVI.4-5, OL, I, 347- $348 ; 10, O L, I, 361)$. La possibilité épistémique rattachée à ces hypothèses ne correspond qu'à une possibilité partielle, insuffisante à garantir qu'il s'agit d'une possibilité réelle. Mais en réalité, il n'y a qu'une unique cause possible. Le statut partiellement conjectural de la physique n'est donc pas incompatible avec la nécessité absolue.

\footnotetext{
${ }^{33}$ Les hypothèses physiques, qui doivent formuler les causes possibles des accidents observables des corps, correspondraient à des possibilités partielles et peuvent ainsi être comprises comme une application de la théorie épistémique des modalités. Elles ne renvoient pas à des possibilités alternatives réelles, mais expriment des possibilités relatives à notre savoir qui dans l'idéal doivent rendre compte d'effets nécessaires par des causes nécessaires. Voir I. Patoluoto, «Hobbes's System of Modalities », in L. Alanen et S. Knuuttila (éds.), Modern Modalities. Studies of the History of Modal Theories from Medieval Nominalism to Logical Positivism, Dordrecht, Kluwer Academic Publishers, 1988, p. 71-84, en particulier p. 79-83.
} 


\section{Leibniz sur la possibilité intrinsèque et l'exigence d'une cause possible}

Selon Leibniz, le rejet du nécessitarisme repose sur la distinction entre la nécessité absolue de la nécessité hypothétique imposée par les causes antécédentes, à condition que l'on considère qu'il y a des individus et des états de choses simplement possibles, quoique inactuels. La réfutation de Hobbes proposée dans la Théodicée ajoute que ces possibles ont eux- mêmes des causes qui, bien qu'inactuelles, n'en sont pas moins possibles. Toutefois le statut de cette affirmation est ambivalent. On peut se demander en effet s'il s'agit d'une simple concession pour les besoins de la réfutation, ou bien s'il s'agit d'un principe constitutif de la possibilité métaphysique selon Leibniz ${ }^{34}$. Or les textes ne semblent pas concorder sur cette question. Dans la Théodicée même, à côté de l'affirmation du $§ 172$ selon laquelle " une chose ne saurait exister, quand une condition requise y manque», d'autres passages suggèrent une formulation plus faible :

En un mot, quand on parle de la possibilité d'une chose, il ne s'agit pas des causes qui doivent faire ou empêcher qu'elle existe actuellement ; autrement on changerait la nature des termes, et on rendrait inutile la distinction entre le possible et l'actuel, comme faisait Abélard et comme Wiclef paraît avoir fait après lui (Théod. § 235).

La possibilité est indépendante de l'existence d'une cause actuelle. Mais il y a une ambiguïté dans l'extrait précédent, quant au fait de savoir si la possibilité requiert en outre la possibilité d'une cause de son existence. Ce passage n'est donc pas incompatible avec le fait que la possibilité d'un événement requière la possibilité d'une cause. Dans d'autres passages, il semble clair que (POS/R) n'est pas un principe de possibilité :

[L]a nécessité et la possibilité, prises métaphysiquement et à la rigueur, dépendent uniquement de cette question, si l'objet en lui-même, ou ce qui lui est opposé, implique contradiction ou non... (Théod. § 367).

\footnotetext{
${ }^{34}$ Cette difficulté est abordée par R. Adams (1994), p. 18-20. Il reconnaît que sur cette question, il y a de la part de Leibniz une hésitation (p. 20). Il propose d'expliquer la discordance des textes à partir de la nature des attributs divins considérés. Il rattache enfin la possibilité interne à la toute-puissance de Dieu. Dans la dernière section, nous critiquerons cette dernière thèse, qui réduit la possibilité intrinsèque à la " puissance objective » des scolastiques.
} 
La possibilité et la nécessité métaphysiques dépendent exclusivement de la nature de l'objet (« l'objet en lui-même »). La non-contradiction interne de cet objet y est érigée en unique critère de possibilité. Le mouvement le plus rapide ou bien un esprit corporel sont par exemple impossibles parce que les éléments qui le composent sont contradictoires ${ }^{35}$. Ainsi, deux conceptions du possible apparaissent dans la Théodicée : le possible comme ce qui est déterminé par la simple non-contradiction d'une part, d'autre part, le possible comme ce qui se rattache à une cause elle-même possible. Loin d'être confinée à un ouvrage tardif qui ne reflète pas toujours les conceptions profondes de Leibniz, cette dualité se retrouve au contraire dans de nombreux autres textes, souvent rédigés au cours des mêmes périodes.

Ainsi, dans une note du De summa rerum de 1676, Leibniz affirme :

L'être absolument parfait ne peut exister, à moins qu'il ne possède une raison d'exister de soi ou par autre chose... Donc ou bien il ne peut avoir nulle raison d'exister et il est ainsi impossible, contre ce que nous avons montré, ou bien il aura [sa raison] de soi, et sera ainsi nécessaire (A VI, 3, 572).

Le raisonnement, ici restreint à l'ens perfectissimum, peut être généralisé. Leibniz rattache en effet la possibilité à l'exigence de raison suffisante appliquée aux possibles : toute chose doit posséder une raison, non seulement de son existence, mais encore de sa possibilité. En conséquence, si les réquisits de $X$ ne peuvent être donnés, non seulement $X$ n'existe pas, mais encore $X$ est impossible. D'autres passages soulignent que la possibilité d'une cause est enveloppée dans la possibilité de chaque état de chose :

[L]es possibilités des individuels ou des vérités contingentes, enferment dans leur notion la possibilité de leurs causes, savoir des décrets libres de Dieu (À Arnauld, 14 juillet 1686, A II, 2, 73).

Afin de concevoir son essence [i.e. l'essence d'une ellipse], le concept d'une cause possible est requis ; mais pour concevoir son existence le concept d'une cause actuelle est requis (À de Volder, 6 juillet 1701, LDV 206/GP II, 225).

\footnotetext{
${ }^{35}$ Voir entre autres, A VI, 3, 463 ; 4-A, 589 et 558 (trad. franç. in Leibniz, Discours de métaphysique. Monadologie, Paris, Gallimard, 2004, p. 288) : «Est impossible ce qui est bien pensable de manière confuse, mais tel que, si on tente de le penser distinctement, on trouvera que les notions dont il est composé répugnent entre elles ou enveloppent une contradiction. Exemples : le mouvement le plus rapide, le plus grand cercle, un esprit corporel ».
} 
Car il suffit qu'un être qui puisse produire la chose, soit possible, afin que la chose soit possible. Généralement parlant, pour qu'un être soit possible, il suffit que sa cause efficiente soit possible; j'excepte la cause efficiente suprême qui doit exister effectivement. Mais c'est ex alio capite, que rien ne serait possible si l'être nécessaire n'existait point. C'est parce que la réalité des possibles et des vérités éternelles doit être fondée dans quelque chose de réel et d'existant (À Bourguet, décembre 1714, GP III, 572).

D'un autre côté, la définition du possible à partir de la non-contradiction conduit Leibniz à distinguer cette possibilité d'une existence possible en tant qu'une telle possibilité peut être causée. Dans un passage des notes de 1678 sur les Opera posthuma de Spinoza, Leibniz souligne cette distinction :

[C]e qui est conçu par soi peut être conçu. Mais on peut encore douter s'il est possible, en la façon que l'on prend ici le terme «possible»; à savoir, non pour ce qui peut être conçu, mais pour ce dont une cause peut être conçue, cause qui peut enfin être résolue dans la cause première. Car tout ce qui peut être conçu par nous ne peut pas pour autant être produit, à cause d'autres choses plus puissantes avec lesquelles il serait incompatible (A VI, 4-B, 1769).

Leibniz distingue ici entre la possibilité déterminée par la concevabilité distincte et la possibilité causale. Il affirme que la première est plus large que la seconde, qui coïncide avec la nécessité hypothétique. Enfin, il reconnaît explicitement que tout possible compris comme concevable n'est pas pour autant susceptible d'être produit. Si quelque chose est concevable mais incompatible avec quelque chose qui possède une plus grande réalité, alors cette chose ne peut être produite. Ceci rejoint la caractérisation du possible per se qui apparaît dans des textes contemporains :

Nous avons défini le possible par sa nature (sua natura possibile), ce qui n'implique pas de contradiction en soi, même si l'on peut dire d'une certaine façon que sa coexistence avec Dieu implique contradiction (...) Je dis ainsi qu'est possible ce dont il y a une essence ou réalité, autrement dit ce qui peut être distinctement conçu (« De libertate et necessitate », A VI, 4-B, 1447).

Ce qui est distinctement concevable est possible, non au sens où la possibilité se réduit à la concevabilité, mais pour autant que ce qui est distinctement concevable correspond à une «essence ou réalité» qui constitue objectivement une possibilité per se. En outre ce qui est possible per se est tel, même s'il est incompatible avec Dieu, l'être nécessaire. Leibniz considère l'exemple de la damnation d'un homme pieux : 
Tout est possible pour Dieu en dehors de ce qui inclut une imperfection. Pécher inclut une imperfection, par exemple la damnation d'un innocent. La damnation d'un innocent est certes possible en soi (in se possibilis), ou n'implique pas contradiction, mais elle n'est pas possible pour Dieu. Au contraire, il semble que la damnation éternelle d'un innocent soit du nombre des choses dont l'existence implique contradiction, mais non l'essence, parce qu'elle peut être parfaitement pensée («De libertate a necessitate in eli- gendo », A VI, 4-B, 1453 ; cf. A VI, 4-B, 1378).

Ce passage, ainsi que la caractérisation du possible per se conduisent à trois remarques. Tout d'abord, (1) le possible per se dépend seulement de la non-contradiction des constituants internes du possible, et il n'y a par exemple aucune contradiction entre la propriété d'être pieux ou vertueux et celle d'être damné ou malheureux ${ }^{36}$. En outre, (2) certains de ces possibles sont impossibles pour Dieu, en ce qu'ils ne peuvent être créés par Dieu, en vertu de sa bonté. En faisant l'hypothèse que ce qui ne peut être actualisé par Dieu ne peut être causé par aucune créature, on est tenté d'inférer enfin (3) que certains possibles per se ne sont les objets d'aucune cause possible, si bien que (POS/R) n'est ni un principe constitutif de la possibilité, ni une thèse vraie de tous les possibles.

Il serait pour le moins surprenant que Leibniz ait défendu, y compris au sein des mêmes ensembles de textes, des conceptions de la possibilité incompatibles entre elles. On s'efforcera de montrer que ces textes se prêtent à une interprétation cohérente du possible. À cet effet, on s'arrêtera au préalable sur deux motifs qui rattachent (POS/R) à un héritage critique à l'égard de Hobbes.

\section{Le rôle des définitions causales et la distinction entre réquisits médiats et immédiats}

L'idée de rattacher la possibilité de l'essence à la causalité doit être lue à la lumière de la théorie de la définition réelle. Une définition réelle se caractérise par le fait qu'elle établit la possibilité de son objet. Or les définitions causales ou génétiques permettent d'établir $a$

\footnotetext{
${ }^{36}$ L'exemple retenu par Leibniz est imparfait, dans la mesure où la propriété d'être damné est une propriété relationnelle, qui dépend du fait que la créature est punie par Dieu.
} 
priori la possibilité de leur objet et sont ainsi une forme privilégiée de définitions réelles ${ }^{37}$. Les définitions causales jouent également un rôle essentiel chez Hobbes et il est possible que la lecture de ce dernier ait influencé Leibniz ${ }^{38}$. Cependant, l'utilité des définitions causales réside précisément dans le fait qu'elles établissent le caractère réel des définitions qui ont pour objets des essences possibles, à l'opposé du conventionnalisme ou de l'ultranominalisme que Leibniz prête à Hobbes ${ }^{39}$. Une définition causale requiert seulement un mode de production possible, autrement dit la formulation d'une hypothèse sur la genèse de l'objet défini, ce qui s'accorde avec la pluralité des modes de production. Une ellipse par exemple peut être conçue comme résultant de la section d'un cône par un plan ou bien comme engendrée par la rotation d'un fil autour de deux foyers dans un plan ${ }^{40}$.

Dans sa correspondance avec De Volder, Leibniz revient sur le rôle des définitions causales, en tant qu'elles nous donnent le concept d'une essence, que cette essence soit générique (comme celle d'une ellipse) ou singulière (comme celle de telle figure ellipsoïdale). L'extrait cité précédemment est en effet une réponse à la thèse de De Volder selon laquelle le concept d'une cause n'est requis que pour concevoir l'existence d'une chose, non pour concevoir son essence. Leibniz affirme que si, afin de concevoir l'existence d'une chose, il faut en effet recourir au concept d'une cause actuelle, afin de concevoir l'essence, il faut recourir au concept d'une cause simplement possible, autrement dit d'un mode de génération. Mais si le concept d'une cause possible est effectivement requis pour concevoir une essence quelconque, cette thèse est susceptible de conduire au nécessitarisme. Michael Griffin développe ainsi les implications potentielles de la réponse à De Volder :

\footnotetext{
${ }^{37}$ A VI, 4-A, 589; DM art.xxiv; A VI, 4-A, 541; TLM 139; A VI, 3, 490-491; A VI, 4-B, 1758. Di Bella (2005), p. 83.

${ }^{38}$ DCo, VI.13, OL, I, 71-72. Hobbes donne l'exemple du cercle défini comme la figure résultant du mouvement d'une ligne droite dans un plan.

${ }^{39}$ Cf. A VI, 4-A, 541 ; TLM 138-139.

${ }^{40}$ TLM 139; cf. A VI, 3, 490, TLM20-21, où Leibniz prend l'exemple d'un carré produit par la réunion de deux triangles rectangles ou bien de deux rectangles.
} 
La seule cause possible, ou fondement suffisant, de l'existence d'une chose est Dieu. Et si l'argument ontologique est correct, l'existence actuelle suit de la possibilité de Dieu. Donc en un sens, le concept qui inclut la possibilité de Dieu inclut également l'existence actuelle de Dieu. Et si Dieu est la raison suffisante de l'existence des choses, alors si l'existence de Dieu est contenue dans leur concept, leur existence actuelle l'est également (Griffin, 2013, 80).

En d'autres termes, la distinction entre une cause actuelle et une cause possible sur laquelle Leibniz fonde son objection au raisonnement de Hobbes est inapplicable lorsque l'on remonte à la cause ultime d'une essence, c'est-à-dire à Dieu comme raison suffisante. Dans la mesure en effet où la simple possibilité de Dieu implique son existence actuelle, il s'ensuit que n'importe quelle essence possible doit être actuelle ${ }^{41}$. Il faut remarquer que Griffin n'adopte pas complètement la conclusion de ce raisonnement, car il estime qu'il y a bien une différence entre une nécessité intrinsèque, liée à l'essence - et de ce point de vue Dieu est unique en ce que son essence enveloppe son existence actuelle - et une nécessité extrinsèque, liée à la série des causes antécédentes ${ }^{42}$. Toutefois, Griffin juge que la distinction entre deux sources de la nécessité, intrinsèque ou extrinsèque, ne change rien à sa force modale, en sorte que Leibniz est au fond contraint de souscrire au nécessitarisme.

Néanmoins cette interprétation, et en particulier l'effondrement de la distinction entre la cause possible et la cause actuelle d'une essence, ne s'impose nullement. En premier lieu, il convient de remarquer que si une définition causale garantit effectivement la possibilité de l'objet défini, cette dernière est cependant constituée en tant que possibilité indépendamment du scénario causal envisagé. La genèse causale est une garantie épistémique a priori de la possibilité de l'objet, de même que l'expérience est une garantie a posteriori, mais elle n'est pas un critère absolu. Pour preuve, on rappellera que Leibniz distingue deux types de définitions réelles: les définitions causales comme on l'a vu, mais également les définitions essentielles qui ont lieu lorsque l'analyse des concepts est poussée jusqu'aux notions primitives correspondant aux « premiers possibles », indépendamment de

\footnotetext{
${ }^{41}$ Selon R. Adams (1994, chap.1, sec.1), le concept d'une chose peut contenir le concept de Dieu comme cause possible, pourvu que ce concept ne contienne pas d'information relative aux décrets actuels portant à l'existence.

${ }^{42}$ Griffin (2013), p. 80.
} 
toute supposition quant au mode de génération ${ }^{43}$. Ainsi la connaissance du mode de génération nous permet de nous assurer a priori de la possibilité de l'objet défini, mais n'est pas exigée pour la possibilité intrinsèque de cet objet, qui dépend de la compatibilité ou non-contradiction de ses éléments constitutifs.

Certes, Leibniz identifie aussi les prima possibilia qui sont les réquisits ultimes des choses, à des causes ou raisons suffisantes de ces choses, autrement dit aux attributs divins :

Cependant je n’oserais pas encore décider, si les hommes pourront jamais instituer une analyse parfaite des notions, c'est-à-dire remonter de leurs pensées aux premiers possibles et aux notions indécomposables, ou, ce qui revient au même, aux attributs absolus de Dieu comme aux causes premières et à l'ultime raison de toutes choses. (A VI, 4-A, 590, trad. P. Schrecker ${ }^{44}$ ).

Les différentes possibilités seraient ainsi constituées à partir de combinaisons de ces réquisits premiers ou premiers possibles qui sont les premières causes des choses. Il semble ainsi que l'on soit reconduit à la position exprimée dans les textes du De summa rerum, qui est elle-même un écho des thèses de Hobbes, à savoir que l'agrégat des réquisits premiers, dont l'énumération forme une définition, ne se trouve que dans l'Être nécessaire ${ }^{45}$. Si, d'une part, les réquisits entrent dans la constitution de l'essence d'une chose et si, d'autre part, ces réquisits sont des causes, alors toute essence est intrinsèquement constituée par le rapport à sa cause.

Il y a néanmoins une inflexion majeure de la part de Leibniz dans sa conception des réquisits, inflexion contemporaine de la définition du vrai par l'inhérence conceptuelle, et qui consiste à définir les réquisits avant tout comme des notions entrant dans la composition d'autres notions $^{46}$. Dans les listes de définitions catégoriales rédigées au début

\footnotetext{
${ }^{43} \mathrm{DM}$ art. xxiv : « et quand elle pousse l'analyse jusqu'à bout jusqu'aux notions primitives, sans rien supposer qui ait besoin de preuve a priori de sa possibilité, la définition est parfaite ou essentielle». Dans les Meditationes de cognitione, veritate et ideis, Leibniz laisse entendre que les définitions causales ne sont qu'une manière de connaître a priori la possibilité de l'objet défini, à côté de l'analyse poussée jusqu'aux prima possibilia qui fournit la connais- sance adéquate ; cf. A VI, 4-A, 589.

${ }^{44}$ Cf. A VI, 4-A, 529 ; C 513 ; TLM 132.

${ }^{45}$ A VI, 3, 587; TLM 30. Sur la définition comme agrégat de réquisits, voir A VI, 3, 133; 573 ; GP III, 247.

${ }^{46}$ A VI, 4-A, 196, TLM 46.
} 
des années 1680, un réquisit est défini comme une condition nécessaire antérieure par nature à ce dont elle est un réquisit ${ }^{47}$. La relation d'antériorité naturelle (natura prius) équivaut à une relation très générale de dépendance qui peut être ou bien épistémique et conceptuelle - et elle est alors définie par un critère de plus ou moins grande facilité à concevoir ou à démontrer - ou bien ontologique ${ }^{48}$. Leibniz ne reviendra plus ni sur cette définition du réquisit ni sur celle de la relation d'antériorité naturelle.

Une fois cette définition fixée, Leibniz introduit une distinction entre les réquisits immédiats et médiats :

Parmi les réquisits, les uns sont médiats, qui doivent être recherchés par la raison, comme les causes ; les autres sont immédiats, comme les parties et les extrémités, et généralement tout ce qui est dans la chose (A VI, 4-A, 627).

Les réquisits médiats correspondent aux causes proprement dites, tandis que les réquisits immédiats sont les éléments qui entrent dans la constitution de la notion de la chose. Un réquisit médiat n'est pas donné par l'analyse conceptuelle, mais doit être recherché par la raison, autrement dit doit rattacher la chose dont il est le réquisit à une série d'entités extérieures à cette chose, selon une structure explicative générale dont la causalité est la forme la plus évidente. La notion de réquisit immédiat définit quant à elle un genre de relations dont l'inhérence constitutive (du point dans la ligne), les relations méréologiques et l'inhérence conceptuelle ou inesse sont différentes espèces. Mais, du point de vue ontologique, c'est la relation de l'inesse qui caractérise les réquisits immédiats :

Les réquisits immédiats sont dans quelque chose (A VI, 4-A, 932).

Être dans quelque chose, ou être inhérent, c'est être un réquisit immédiat (A VI, 4-A, 941).

A est en B si toute chose qui est immédiatement requise pour A est aussi requise immédiatement pour B. Ce qui est immédiatement requis pour quelque chose, de sorte que rien de plus ne soit

\footnotetext{
${ }^{47}$ A VI, 4-A, 389; 402; 932; A VI, 4-B, 1413; C 417.

${ }^{48}$ A VI, 4-A, 180; 402. Cf. J.-B. Rauzy, « Quid sit natura prius? La conception leibnizienne de l'ordre », Revue de métaphysique et de morale 100 (1995), p. 31-48 ; S. Di Bella (2005), p. 246 sq. ; « Leibniz on Causation : Efficiency, Explanation and Conceptual Dependence », Quaestio 2 (2002), p. 411-447.
} 
immédiatement, ni même médiatement, requis pour elle, pourra être appelé la réalité (A VI, 4, 990; TLM 388) ${ }^{49}$.

Ainsi les réquisits immédiats sont réellement inhérents dans la chose dont ils sont les réquisits. En outre, les réquisits immédiats fondamentaux sont constitutifs de la réalité de cette chose, par quoi il faut comprendre qu'ils constituent l'essence de la chose en question. Ainsi la réalité du cercle est donnée par les propriétés fondamentales du cercle, à partir desquelles peuvent se déduire l'ensemble des autres propriétés. Dans la correspondance avec Arnauld, Leibniz nomme " prédicats primitifs » d'une substance individuelle tous ceux qui ne dépendent d'aucun autre. Ces prédicats primitifs sont des dénominations intrinsèques de cette substance individuelle. En outre, la totalité des prédicats primitifs suffit à former une notion complète, à partir de laquelle il est possible de rendre raison de la totalité des prédicats attribués à un individu ${ }^{50}$. Les relations causales de cette substance individuelle avec les autres choses ne font pas partie de ces prédicats primitifs. Néanmoins, elles sont également contenues dans la notion de cette chose, comme en général tous les réquisits médiats de cette chose. Cette relation des réquisits médiats à la substance individuelle n'est pas une relation d'inhérence, mais une relation distincte, à laquelle Leibniz donne le nom d'indigentia :

Donc une modification requiert autre chose que le besoin (indigentia) du concept d'une autre chose et l'inhérence (inesse) (qui est commune aux propriétés et aux modes), est davantage que le fait d'avoir besoin du concept d'une autre chose. Mon opinion est qu'il n'y a rien dans la totalité des créa- tures qui n'ait besoin (indigeat) pour son concept parfait du concept de n'importe quelle autre chose dans la totalité des choses, puisque chaque chose exerce une influence (influat) sur n'importe quelle autre, en sorte que si on feignait qu'elle était supprimée ou différente, toutes les choses dans le monde seraient différentes de ce qu'elles sont maintenant (À de Volder, 6 juillet 1701, GP II, 226).

\footnotetext{
${ }^{49}$ Voir S. Di Bella, « Leibniz et l'inhérence », Archives de Philosophie 77 (2014), p.17-42, en particulier p. 23-28.

${ }^{50}$ A II, 2, 50 : «Car tous les predicats d'Adam dependent d'autres predicats du même Adam, ou n'en dependent point. Mettant donc à part ceux qui dependent d'autres, on n'a qu'à prendre ensemble tous les predicats primitifs, pour former la notion complete d'Adam suffisante à en deduire tout ce qui luy doit jamais arriver, autant qu'il faut pour en pouvoir rendre raison ».
} 
De même qu'il est possible d'associer à l'ensemble des prédicats primitifs une notion complète que l'on peut caractériser comme une notion étroite, de même est-il possible d'associer à ce dont dépend cette substance, en particulier dans un sens causal, un ensemble de prédicats constituant la notion complète de cette substance au sens large, ensemble qui inclut la référence à la totalité des états des autres substances, en vertu de la connexion des choses et de l'expression universelle. Cependant cet ensemble n'est pas inhérent à la substance. Certes, sa notion complète en a besoin ou la requiert, mais la nécessité de cette connexion est seulement hypothétique et fondée sur des décrets divins, mais non absolue et conceptuelle ${ }^{51}$.

\section{La possibilité per se comme possibilité métaphysique}

Ces distinctions permettent de résoudre le problème des textes discordants évoqués dans la section 5. La possibilité est constituée par la compatibilité des réquisits immédiats ou éléments constitutifs et qui répond au concept étroit de ces substances (ou bien au concept étroit d'un individu vague). La possibilité dépend seulement de ce que Leibniz caractérise dans la Théodicée comme " l'objet en lui-même » (§ 367) et le principe (POS/R) est inopérant à ce niveau.

Mais si l'on considère le concept large, il apparaît que chacun de ces cas enveloppe des réquisits médiats déterminés en sorte que la possibilité de la cause est supposée, laquelle coïncide avec une partie de ce dont a besoin (indiget) la substance en question pour que son existence ou tel état soit possible. C'est dans ce sens que Leibniz peut affirmer en particulier que les possibilités individuelles enferment des décrets divins possibles qui en sont les causes.

Certains cas demeurent cependant problématiques, en particulier celui de la damnation d'un innocent. N'étant pas intrinsèquement contradictoire, un tel état de choses est une possibilité en soi. Mais nulle cause n'en peut être donnée, puisqu'il faudrait pour cela que Dieu manque de bonté, ce qui est métaphysiquement impossible. Plus généralement, tout état de choses ne figurant pas dans le meilleur des mondes possibles dérogerait à la

\footnotetext{
${ }^{51}$ C'est une conséquence de la séparabilité des substances. Cf. GP II, 307 ; 496.
} 
perfection divine et impliquerait une contradiction, non en lui-même, mais en considération de la perfection divine. Il ne semble donc pas possible de rattacher cet état de choses à sa cause première de sorte que, s'il est intrinsèquement possible, il n'en est pas moins extrinsèquement et métaphysiquement impossible.

On ne saurait pleinement résoudre cette dernière difficulté dans les limites de cette étude. Il suffira de considérer en conclusion ce qui sépare Leibniz de Hobbes dans la conception des modalités, et plus précisément du rapport entre possibilité et pouvoir. Il faut en premier lieu rappeler le rapport étroit entre la notion de décret possible et celle de loi. En effet, un décret possible désigne une loi par laquelle les états d'une substance s'enchaînent entre eux et s'accommodent avec les états des autres substances dans un monde possible. Les décrets possibles ne renvoient donc pas à des décisions que Dieu pourrait prendre étant donné sa nature, et en particulier sa sagesse et bonté qui forment sa perfection. Et de ce point de vue, toute collection de possibles peut être rattachée à une loi qui est un décret possible divin ${ }^{52}$. Ces décrets sont inscrits dans les notions complètes des possibles et sont des conditions pour qu'une possibilité individuelle soit représentée comme une possibilité. Mais cela n'implique pas que Dieu possède en effet la puissance d'actualiser un tel possible, ou encore que tout possible soit créable.

La distinction scolastique, telle que la théorise Duns Scot, entre possibilité logique et puissance objective (potentia objectiva) permet d'éclairer ce point. Selon Duns Scot, quelque chose est en puissance objective si cette chose n'existe pas mais peut exister comme objet ou terme d'un pouvoir ${ }^{53}$. Cette puissance objective se distingue de la possibilité logique qui lui est antérieure et quelque chose est un être possible pourvu que son existence n'implique pas contradiction ${ }^{54}$. Duns Scot donne comme exemple la possibilité de l'existence du monde, qui subsiste même dans l'hypothèse impossible où

\footnotetext{
${ }^{52}$ Cf. DM art. vi et A VI, 4-B, 1619.

${ }^{53}$ J. Duns Scot, Quaestiones in Metaphysicam IX, q. 1-2, n. 39sq. Opera Philosophica IV, 523-526.

${ }^{54}$ J. Duns Scot, Ordinatio I, d.36, q. un., n.61, éd. C. Balic et al., Rome, Ex typis Vaticanis, 1953, vol. VI, p. 296 : « illam possibilitatem [logicam] consequitur possibilitas obiectiva, et hoc supposita omnipotentia Dei quae respicit omne possibile (dummodo illud sit aliud a se), tamen illa possibilitas logica, absolute - ratione sui - posset stare, licet per impossibile nulla omnipotentia eam respiceret.»
} 
Dieu ne serait pas tout-puissant et où il n'y aurait donc pas de cause créatrice du monde ${ }^{55}$. L'être possible, son statut ontologique, est ainsi indépendant du rapport à une puissance active. Ce qui est possible est en effet formellement possible par lui-même, en vertu de sa non-contradiction (non repugnantia). Son statut ontologique est celui d'un objet de pensée, produit dans un être intelligible par l'intellect divin. Pour autant, la toute-puissance ne constitue pas la réalité du possible, ce qui n'exclut donc pas qu'il y ait du possible qui ne soit pas un objet de la toute-puissance divine ${ }^{56}$.

Leibniz exploite une possibilité théorique semblable dans le cas du possible per se. Ainsi, (1) la possibilité intrinsèque d'un innocent damné est constituée en possibilité logique, parce qu'il n'y a pas d'incompatibilité intrinsèque entre le fait d'être juste et celui d'être malheureux. En outre, (2) relativement à l'attribut de la toute-puissance divine, cet état de choses est une possibilité objective, parce qu'il y a une loi ou un décret possible par lequel Dieu pourrait récompenser la vertu par la damnation, par quoi cette possibilité intrinsèque peut s'inscrire dans un ordre des choses ou un monde possible. Cependant, (3) si l'on tient compte de la sagesse et bonté de Dieu, il ne s'agit pas d'une possibilité objective : un monde contenant un tel état de choses est en effet inactualisable par Dieu ${ }^{57}$. En ce sens seulement, il est possible de dire que l'existence d'un tel monde implique contradiction,

\footnotetext{
55 J. Duns Scot, Ordinatio I, d.7, q.1, n.27, Vat. IV, 118-119. Cf. Peter King, « Duns Scotus on Possibilities, Powers, and the Possible », in Kneepkens et Lorenz (2001), p. 175-199, p. 194.

${ }^{56}$ J. Duns Scot, Ordinatio I, d.43, q.un, n.6, Vat. VI, 354 : « Lapis est possibilis ex se formaliter ; ergo et reducendo quasi ad primum extrinsecum principium intellectus divinus erit illud a quo est prima ratio possibilitatis in lapide. Non ergo illa potentia activa a qua Deus dicitur omnipotens, est prima ratio possibilitatis in lapide». Dans la tradition thomiste au contraire, le discours sur le possible est entièrement réductible à un discours sur la puissance divine. Cf. Thomas d'Aquin, Sum. Theol. I, q. 9, a. 2, in corp. : « Omnes enim creaturae, antequam essent, non erant possibiles esse per aliquam potentiam creatam, cum nullum creatum sit aeternum : sed per solam potentiam divinam, inquantum Deus poterat eas in esse producere ». Arnauld s'inscrit clairement dans la tradition thomiste ; cf. sa lettre à Leibniz du 13 mai 1686, A II, 2, 36-37 : «tout ce que nous appellons substances possibles purement possibles, ne peut estre autre chose que la toute puissance de Dieu».

${ }^{57}$ R. Adams (1994, p.20) suggère une interprétation similaire dans laquelle la distinction entre les niveaux de possibilité se rapporte aux différents attributs divins envisagés, sa toute-puissance ou bien sa bonté.
} 
sans pourtant que son essence n'implique contradiction. Enfin, (4) la possibilité logique d'un innocent damné, comme toutes les vérités concernant les possibles et les essences sur lesquelles elles sont fondées subsiste dans la région des vérités éternelles. À ce titre, il faut dire qu'elle se rapporte nécessairement à un fondement actuel, et non à une simple cause possible $^{58}$. Cependant, pour reprendre la formule de Leibniz à Bourguet, c'est ex alio capite : en d'autres termes, cela ne revient pas à l'exigence hobbesienne, puisque l'exigence d'un fondement actuel s'impose seulement pour autant que toute possibilité logique requiert un fondement ontologique actuel, qui est l'entendement divin ou la région des vérités éternelles.

\section{Conclusion}

L'étude du rapport de Leibniz aux conceptions modales de Hobbes a permis de mettre en lumière la nature des réquisits des possibles inactuels, dont le rôle est crucial dans la réfutation du nécessitarisme. Nous avons ainsi vu que Hobbes souscrivait à une conception extrêmement stricte du possible, nécessairement fondé dans une puissance, laquelle ne définit une possibilité réelle que lorsque l'ensemble des réquisits d'un état de choses est présent, ce qui le conduit à identifier le possible à l'actuel. Les textes de Leibniz présentent quant à eux une ambivalence, tantôt faisant de la possibilité d'une cause un principe de possibilité, tantôt au contraire paraissant fonder la possibilité dans la simple noncontradiction interne. Nous avons essayé d'établir que cet écart ne relève pas d'une distinction entre le possible dans un sens causal et le possible dans un sens logique, comme si ce dernier ne définissait pas une possibilité réelle, métaphysique. Nous avons essayé de réduire l'ambivalence des textes leibniziens en la rattachant à une distinction entre, d'une part, la relation métaphysique de l'inhérence et, d'autre part, la relation expressive ou représentative de l'indigentia. Selon la première, la possibilité dépend exclusivement de la non-contradiction interne des réquisits immédiats. Selon la seconde, tout possible renvoie à titre de réquisits médiats aux causes en vertu desquelles il se produit dans tel monde et, en

\footnotetext{
${ }^{58}$ Cf. Monadologie §§ 43-44; A VI, 4-B, 1599-1600; 1635; Grua 392-393; GP VII, 305; Théodicée §§ 20 ; $184 ; 189$; GM III, 586.
} 
raison de la connexion universelle, à l'ensemble des possibles figurant dans le même monde. Enfin, le type de possibilité définie par la première s'oppose à la nécessité absolue, tandis que la seconde relève de la nécessité hypothétique. 